\title{
Assessment of Burden of COPD (ABC) tool: perception versus evidence
}

\author{
Sarah Houben-Wilke ${ }^{1}$, Frits M.E. Franssen ${ }^{2}$, Ineke Kok ${ }^{3}$, Henk J. Otten ${ }^{4}$, \\ Johan P. Wempe ${ }^{5}$ and Jan H. Vercoulen ${ }^{6}$ \\ Affiliations: 'Dutch Lung Centers, Horn, The Netherlands. ${ }^{2} \mathrm{CIRO}$, Horn, The Netherlands. ${ }^{3}$ Merem, Hilversum, \\ The Netherlands. " Revant, Pulmonary Rehabilitation Center Schoondonck, Breda, The Netherlands. \\ ${ }^{5}$ University Medical Center Groningen, Groningen, The Netherlands. ${ }^{6}$ Radboud University Medical Center, \\ Nijmegen, The Netherlands.
}

Correspondence: Sarah Houben-Wilke, Hornerheide 1, 6085 NM Horn, The Netherlands.

E-mail: sarahwilkedciro-horn.nl

@ERSpublications

Multidimensionality should be assessed by studying multi-dimensions, not by using a multidimensional assessment tool http://ow.ly/ht1c3091F29

Cite this article as: Houben-Wilke S, Franssen FME, Kok I, et al. Assessment of Burden of COPD (ABC) tool: perception versus evidence. Eur Respir J 2017; 49: 1602382 [https://doi.org/10.1183/13993003.02382-2016].

\section{Drawing the context}

It is not disputable that chronic obstructive pulmonary disease (COPD) is a multifaceted disease affecting patients' health beyond the lungs with immense variability between individuals [1-3]. Given that complexity and variability, multidimensional assessment and treatment integrating concepts of complex adaptive systems are needed [4]. Agusti and MacNee [5] stated that physicians need a "control panel" including at least three dimensions (severity, activity and impact) to assess different elements of the disease. This "control panel" might be useful for routine clinical practice and to move COPD management towards personalised medicine [5]. However, can one "control panel" reflect the complexity and heterogeneity of this complex, multisystem disease? Can one tool guarantee a personalised approach?

\section{Multidimensional indices}

During the previous 10 years, several multidimensional assessment tools have been developed trying to capture the multicomponent nature of COPD, e.g. the ADO (age, dyspnoea, airflow obstruction) index [6], the BODE (body mass index, airflow obstruction, dyspnoea and exercise) index [7] as well as several BODE modifications (e.g. i-BODE [8]), the DOSE (dyspnoea, obstruction, smoking, exacerbation) index [9] or the HADO (health, activity, dyspnoea, obstruction) index [10]. The performance and usefulness of these indices have been summarised before [11].

Remarkably, these indices mainly focus on medical, pathophysiological and/or symptomatic parameters and have been developed as prognostic indices for COPD. Multidimensional measures including patient-reported outcomes are scarce. Typically, a patient-reported outcome is "any report of the status of a patient's health condition that comes directly from the patient, without interpretation of the patient's response by a clinician or anyone else" [12]. One important patient-reported outcome is health status, which can be defined as "the impact of health on a person's ability to perform and derive fulfilment from the activities of daily life" [13]. As already stated by Jones [14], "health status measurement (...) is a process that is essentially similar to a highly structured clinical history (...)" emphasising health status as an important patient-reported outcome in the assessment of COPD severity [15]. Indeed, health status has been shown to be associated with exacerbations, hospitalisations and dying [16].

Received: Dec 052016 | Accepted: Dec 132016

Conflict of interest: None declared.

Copyright @ERS 2017 


\section{Assessment of burden of COPD (ABC) tool}

In 2014, the Assessment of Burden of COPD (ABC) tool was developed combining the patient-reported ABC scale (based on the Clinical COPD Questionnaire [17], consisting of five domains: symptoms, functional state, mental state, emotions and fatigue) with smoking status, exacerbations, dyspnoea, body mass index, lung function and self-reported physical activity [18]. The $\mathrm{ABC}$ tool is a computer program visualising these outcomes using coloured balloons (figure 1) [19]. It was originally developed to "assess and visualize the integrated health status of patients with COPD, and to provide patients and healthcare providers with a treatment algorithm" [20]. The ABC tool has recently been evaluated in a randomised control trial in primary and secondary care settings in the Netherlands studying the effectiveness compared with usual care on health status in the management of patients with COPD over a period of 18 months [19]. The primary outcome was health status as assessed with the St George's Respiratory Questionnaire (SGRQ). The secondary outcomes were health status as assessed by the COPD Assessment Test (CAT) and Patient Assessment of Chronic Illness Care (PACIC, a measure for perceived quality of care).

\section{Perception versus evidence}

In the light of future healthcare encouraging a patient's functionality and independency, adaptability and flexibility as well as control, autonomy and self-management [21], the authors' hypothesis that "giving patients the possibility and the responsibility in setting personal treatment goals and making their own treatment plan will influence self-management, facilitate and stimulating behavioural change, and eventually lead to an improved quality of life" (by using the ABC tool) sounds promising [19]. However, there are several shortcomings that should be addressed.

First, there are some concerns with regards to data analyses and interpretation. The authors found that $33.4 \%$ of the patients in the intervention group had a clinically relevant improved health status after 18 months compared with $22.3 \%$ patients in the control group. However, the mean health status scores did not change or differ between groups. Furthermore, the proportion of patients with a deterioration in health status was not statistically significant after 18 months. In addition, almost $70 \%$ of the intervention group (as well as almost $80 \%$ of the control group) did not show an improvement in health status challenging the clinical relevance of these findings. Surprisingly, CAT scores did not differ between treatment groups after 18 months. However, the authors did not provide the mean CAT scores (for each group) or information about the proportion of patients with a clinically relevant improvement in CAT score (consistently with SGRQ). Furthermore, changes in PACIC score were rather small and information on the clinical relevance has not been provided. Additionally, differences in baseline patient characteristics between the intervention group and the control group have not been tested statistically.

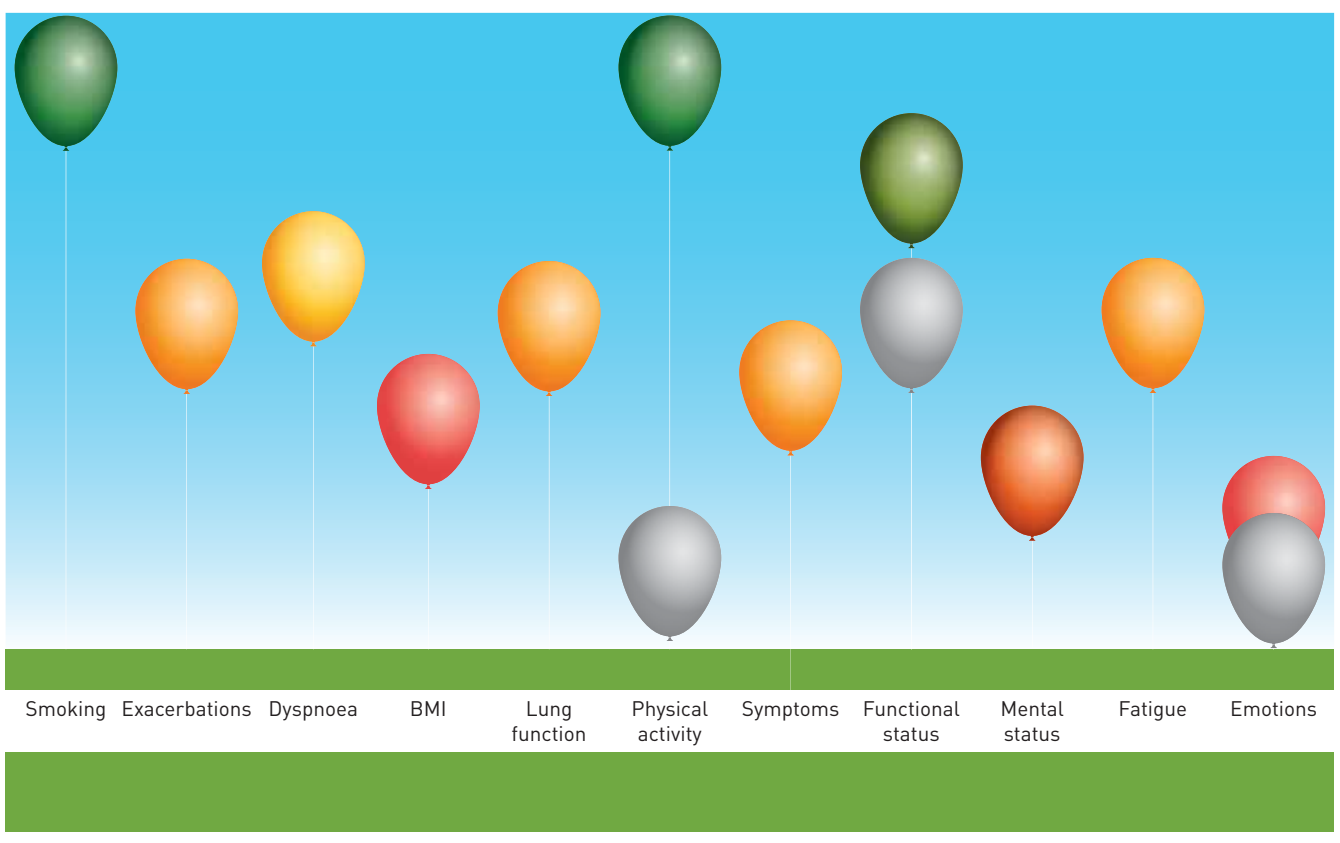

FIGURE 1 Visualisation according to the Assessment of Burden of COPD (ABC) tool; green balloons indicate a satisfactory score, red balloons a low score and orange balloons an intermediate score. Grey balloons represent the balloons of previous visits. Reproduced with permission [19]. 
Second, the rationale, hypothesis, objectives and methodology seem incoherent. The ABC assessment tool was originally developed to "assess and visualize the integrated health status of patients with COPD, and to provide patients and healthcare providers with a treatment algorithm" [20]. Further, the authors mention that the $\mathrm{ABC}$ tool can be used as a communication tool in primary and hospital care. On the other hand "it provides the opportunity to support personalized care planning" [19]. The study protocol stated that "it has been developed to classify patients, but also to provide visual insight into the burden of COPD and to provide treatment advice" [20]. The authors hypothesised that the ABC tool “... will influence self-management, facilitate and stimulate behaviour change ...” [19]. However, the primary aim of this study was to assess the effectiveness of the ABC tool in patients with COPD on health status. Thus, the rationale and aim of the $\mathrm{ABC}$ tool remain unclear and should have been clarified beforehand. Has this instrument been developed to support and improve communication? Or to encourage personalised care planning? Why did the authors choose health status as the primary outcome? Given the fact that the $\mathrm{ABC}$ tool is based on the Clinical COPD questionnaire, how is this instrument related to other health status instruments as the $\mathrm{ABC}$ tool is defined as an instrument to assess the burden of COPD?

Third, the authors conclude that patients "treated with the $\mathrm{ABC}$ tool were more likely to report clinically relevant improvement in quality of life" [19]. However, the underlying mechanisms or reasons of this improvement remain unclear and argumentation on this point is lacking. Did the health status of these patients improve because of an improvement in their perceived quality of care? Correlations between health status (SGRQ) and perceived quality of care (PACIC) have not been reported. Or did the patients simply score better because the ABC tool was used (participation bias)? Or is the improvement a consequence of "personalised" treatment effects? Did patients with an improved health status improve on specific domains of the $\mathrm{ABC}$ tool? The authors described that healthcare providers were not stimulated to practise using the tool, which may also cause some variability. Finally, the wording "treated with the ABC tool" might be misleading. Thus, some questions remain unanswered but play a crucial role in understanding the use and impact of this measure.

Fourth, one important component is missing in this multidimensional index: the assessment of comorbidities. If one of the aims is "to provide visual insight into the burden of COPD", comorbidities cannot be ignored. Comorbidities play a major role in the burden and management of COPD [22] but have not been included in the ABC tool by any means at all.

Fifth, there are some concerns about the psychometric quality of the $\mathrm{ABC}$ tool. It is assumed that the instrument contains five domains, each reflecting a conceptually distinct concept. However, this has not been tested empirically. In addition, the coloured balloons suggest the presence of normative data that discriminate for each domain between normal functioning, mild problems and severe problems. No information is provided on how these norms are determined: clinical experience, empirical data or otherwise?

Finally, do we need another (multidimensional) tool to assess health status? This might be more confusing than providing a practical guide for health status assessment in clinical practice (and research). Evaluating the psychometric properties of existing instruments may better contribute to a progression in the field of health status measurement [23].

In summary, the development, validation and effectiveness of the $\mathrm{ABC}$ tool lacks consistency and evidence. It might be a useful communication tool (understandable for patients in primary and secondary care) to involve patients in their disease management. Unfortunately, the simplicity of the balloons masks the lack of scientific and clinical evidence. COPD should be multidimensionally assessed by rather assessing multidimensions than using one multidimensional assessment tool. With regards to future healthcare, one tool cannot provide a patient profile enabling tailored, individualised treatment. Indeed, healthcare providers involved in the management of COPD must be encouraged to target the unique needs and abilities reflecting the individual complexity of the patient. With regards to this $\mathrm{ABC}$ tool, perception overwhelms evidence.

\section{References}

1 Vanfleteren LE, Spruit MA, Groenen M, et al. Clusters of comorbidities based on validated objective measurements and systemic inflammation in patients with chronic obstructive pulmonary disease. Am J Respir Crit Care Med 2013; 187: 728-735.

2 Divo MJ, Casanova C, Marin JM, et al. COPD comorbidities network. Eur Respir J 2015; 46: 640-650.

3 Spruit MA, Augustin IM, Vanfleteren LE, et al. Differential response to pulmonary rehabilitation in COPD: multidimensional profiling. Eur Respir J 2015; 46: 1625-1635.

4 Wouters EF, Augustin IM. COPD health-care delivery: a holistic and dynamic approach is needed. Lancet Respir Med 2016; 4: e30-e31.

5 Agusti A, MacNee W. The COPD control panel: towards personalised medicine in COPD. Thorax 2013; 68: 687-690.

6 Puhan MA, Garcia-Aymerich J, Frey M, et al. Expansion of the prognostic assessment of patients with chronic obstructive pulmonary disease: the updated BODE index and the ADO index. Lancet 2009; 374: 704-711. 
7 Celli BR, Cote CG, Marin JM, et al. The body-mass index, airflow obstruction, dyspnea, and exercise capacity index in chronic obstructive pulmonary disease. N Engl J Med 2004; 350: 1005-1012.

8 Williams JE, Green RH, Warrington V, et al. Development of the i-BODE: validation of the incremental shuttle walking test within the BODE index. Respir Med 2012; 106: 390-396.

9 Jones RC, Donaldson GC, Chavannes NH, et al. Derivation and validation of a composite index of severity in chronic obstructive pulmonary disease: the DOSE Index. Am J Respir Crit Care Med 2009; 180: 1189-1195.

10 Esteban C, Quintana JM, Aburto $\mathrm{M}$, et al. A simple score for assessing stable chronic obstructive pulmonary disease. QJM 2006; 99: 751-759.

11 Dijk WD, Bemt L, Haak-Rongen S, et al. Multidimensional prognostic indices for use in COPD patient care. A systematic review. Respir Res 2011; 12: 151.

12 US Food and Drug Administration. Guidance for Industry Patient-reported Outcome Measures: Use in Medical Product Development to Support Labeling Claims. US Department of Health and Human Services, Food and Drug Administration; Center for Drug Evaluation and Research (CDER); Center for Biologics Evaluation and Research (CBER); Center for Devices and Radiological Health (CDRH), 2009. Available from: www.fda.gov/ downloads/Drugs/GuidanceComplianceRegulatoryInformation/Guidances/UCM193282.pdf

13 Curtis JR, Patrick DL. The assessment of health status among patients with COPD. Eur Respir J 2003; 41: 36s-45s.

14 Jones PW. Health status measurement in chronic obstructive pulmonary disease. Thorax 2001; 56: 880-887.

15 Vestbo J, Hurd SS, Agustí AG, et al. Global strategy for the diagnosis, management, and prevention of chronic obstructive pulmonary disease: GOLD executive summary. Am J Respir Crit Care Med 2013; 187: 347-365.

16 Wilke S, Jones PW, Müllerova $\mathrm{H}$, et al. One-year change in health status and subsequent outcomes in COPD. Thorax 2015; 70: 420-425.

17 van der Molen T, Willemse BW, Schokker S, et al. Development, validity and responsiveness of the Clinical COPD Questionnaire. Health Qual Life Out 2003; 1: 13.

18 Slok $\mathrm{AH}$, in 't Veen JC, Chavannes $\mathrm{NH}$, et al. Development of the Assessment of Burden of COPD tool: an integrated tool to measure the burden of COPD. NPJ Prim Care Respir Med 2014; $24: 14021$.

19 Slok AH, Kotz D, van Breukelen G, et al. Effectiveness of the Assessment of Burden of COPD (ABC) tool on health-related quality of life in patients with COPD: a cluster randomised controlled trial in primary and hospital care. BMJ Open 2016; 6: e011519.

20 Slok AH, In 't Veen JC, Chavannes NH, et al. Effectiveness of the Assessment of Burden of Chronic Obstructive Pulmonary Disease $(\mathrm{ABC})$ tool: study protocol of a cluster randomised trial in primary and secondary care. BMC Pulm Med 2014; 14: 131.

21 Kaljouw M, van Vliet K. Naar nieuwe zorg en zorgberoepen: de contouren. Diemen, Zorginstituut Nederland, 2015.

22 Vanfleteren LE, Spruit MA, Wouters EF, et al. Management of chronic obstructive pulmonary disease beyond the lungs. Lancet Respir Med 2016; 4: 911-924.

23 Weldam SW, Schuurmans MJ, Liu R, et al. Evaluation of Quality of Life instruments for use in COPD care and research: A systematic review. Int J Nurs Stud 2013; 50: 688-707. 